\title{
Kondo physics in artificial molecules
}

\author{
K.Kikoin ${ }^{1}$ \\ Department of Physics, Ben-Gurion University, Beer Sheva 84105, Israel \\ Y. Avishai ${ }^{1,2}$ \\ ${ }^{1}$ Department of Physics, Ben-Gurion University, Beer Sheva 84105, Israel \\ 2 Department of Applied Physics, University of Tokyo
}

\begin{abstract}
Recent advancement in fabrication technologies enable the construction of nano-objects with rather rich internal structures such as double or triple quantum dots, which can then be regarded as artificial molecules. The main new ingredient in the study of the Kondo effect in such artificial (and also in natural) molecules is the internal symmetry of the nano-object, which proves to play a crucial role in the construction of the effective exchange Hamiltonian. This internal symmetry combines continuous spin symmetry $S U(2)$ and discrete point symmetry (such as mirror reflections for double dots or discrete $C_{3 v}$ rotation for equilateral triangular dots. When these artificial molecules are attached to metallic leads, the set of dot operators appearing in the effective exchange Hamiltonian generate a group which is refereed to as the dynamical symmetry group of the system dot-leads [mostly $S O(n)$ or $S U(n)$ ], and the pertinent group parameters (such as the value of $n$ ) can be controlled by experiment. In this short review we clarify and expand these concepts and discuss some specific examples. In particular we concentrate on the difference between the chain geometry and the ring geometry. When a perpendicular magnetic field is applied in the ring geometry, its gauge symmetry $U(1)$ is involved in the interplay with the spin and orbital dynamics of the dot.
\end{abstract}




\section{INTRODUCTION: KONDO MAPPING AND BEYOND}

There are numerous models in the literature of condensed matter theory, whose significance for achieving progress in our understanding of Nature goes far beyond the original aim of explaining specific experimental observations. One may mention in this context the Bardeen-Cooper-Schrieffer's explanation of the nature of electron pairing in superconductors, the Ginzburg-Landau equation intended for describing critical fluctuations, the concept of self-localization of excitations in a perfect crystal formulated by Deigen, Pekar and Toyozawa and various other seminal ideas. The explanation offered by J. Kondo for the puzzling shallow minimum in the temperature dependent resistivity of metals doped by magnetic impurities [1] is one of the most salient examples of this kind of this scenarios. To explain it, consider first Kondo original idea, which was formulated within the framework of a well established Hamiltonian describing exchange interaction between an impurity spin $\mathbf{S}_{\mathbf{r}}$ located on a given site $\mathbf{r}$ and the spin density $\mathbf{s}_{\mathbf{r}}$ pertaining to a Fermi sea of conduction electrons at this site. The latter is defined by the Fourier transform of the itinerant spin $\mathbf{S}_{\mathbf{k} \mathbf{k}^{\prime}}=c_{\mathbf{k} \sigma}^{\dagger} \hat{\tau} c_{\mathbf{k}^{\prime} \sigma^{\prime}}$ projected on the impurity site $\mathbf{r}$, namely $\mathbf{s}_{\mathbf{r}}=\sum_{\mathbf{k k}^{\prime}} \mathbf{s}_{\mathbf{k k}^{\prime}} \exp \mathrm{i}\left(\mathbf{k}-\mathbf{k}^{\prime}\right) \cdot \mathbf{r}$. Here $\hat{\tau}$ is the vector of Pauli matrices for a spin $1 / 2$. The so called $s d$-exchange Hamiltonian is,

$$
H_{\mathrm{sd}}=\sum_{\mathbf{k}, \sigma} \varepsilon_{k} c_{\mathbf{k} \sigma}^{\dagger} c_{\mathbf{k} \sigma}+J \mathbf{S}_{\mathbf{r}} \cdot \mathbf{S}_{\mathbf{r}}
$$

where $\varepsilon_{k}$ is the energy dispersion of the itinerant electrons and $J$ is the exchange coupling constant. At first glance, it looks deceptively simple. However, Kondo noticed that the first correction to the impurity scattering amplitude of the conduction electrons beyond the Born approximation suffers an infrared logarithmic divergence in energy or temperature. This results in a singular behavior of amplitudes for an antiferromagnetic sign of the exchange coupling (that is, $J>0$ ), and renders perturbation theory inapplicable below a certain energy scale known as the Kondo temperature. Nearly two decades of incessant efforts to take this singularity properly into account and to find the ground state of the system had crowned with finding the exact solution both numerically (in a framework of Numerical Renormalization Group, NRG) [2] and analytically (by using the Bethe ansatz) [3, 4].

Soon after Kondo's breakthrough, theoreticians started to extend this promising conceptual framework for other physical situations and for more complex objects than simple localized moments. It was recognized that the Kondo mechanism should work also in sys- 
tems exhibiting electron tunneling, where two metallic slabs are separated by thin dielectric layer, which forms a tunnel barrier for electrons moving from one slab to the other. It was shown [5, 6] that the magnetic impurity located somewhere near the tunneling layer plays the same role in tunnel conductance as magnetic impurity immersed in a metal (and subject to exchange interaction with Fermi sea electrons) does for impurity resistance. It was also shown [7] that the Friedel-Anderson model [8, 9] for resonance scattering of conduction electrons by the electrons occupying the $3 d$ levels of transition metal impurities can be mapped on the exchange Hamiltonian (11) provided the strong Coulomb interaction in the $3 D$ shell suppresses charge fluctuations on the impurity site. In the next stage of development orbital degrees of freedom were incorporated in the Kondo physics. The idea of this generalization is based on the fact that the magnetic impurity being put in the center of coordinates imposes its point symmetry on the otherwise translationally invariant crystal, and the appropriate description of scattered waves should exploit the formalism of partial wave expansion (either in spherical waves [8] or in cubic harmonics [10]). Based on this idea, the generalized Schrieffer-Wolff model was proposed [11, 12], where the magnetic impurity is described as an effective $N$-component moment, but the exchange scattering is not restricted by the usual spin selection rule $\Delta m=0, \pm 1$ for the projection $m$ of this moment. Another version of this model allots the impurity and band states both by spin and orbital (referred to as "color" in the general case) index. In case of spin $s=1 / 2$ and $N$ colors the symmetry of the impurity is $S U(2 N)$ [13, 14]. Appearance of additional degrees of freedom in the Kondo Hamiltonian might lead to the interesting scenario of overscreened Kondo effect, which arises when the number of conduction electron "colors" exceed that of impurity moment [15]. This effect is characterized by the non-Fermi-liquid low-temperature thermodynamics unlike the standard Kondo effect, which only modifies (although radically)) the Fermi liquid properties of undoped metal [16].

Another direction of expanding the Kondo physics is realized in mapping the Kondo or Anderson Hamiltonian on those of other (not necessarily magnetic) systems. A great variety of such generalizations is possible because some quantum systems may be described by a pseudospin, provided their low-energy states are characterized as an effective twolevel system (TLS) and external perturbations allow transitions between these levels. The first example of such generalization was suggested by the Hamiltonian describing tunneling between a Fermi sea electrons and an atom sitting in a double-well potential [17]. Another 
possibility of this sort arises when the crystal field splitting is involved in formation of the low-energy states of impurity atom (quadrupolar Kondo effect) [18, 19]. This type of "exotic" Kondo system was surveyed in a detailed review [20]. One should mention also the possibility of involving orbital degrees of freedom in the formation of Kondo-resonance for an adsorbed transition metal impurity, where the orbital degeneracy is lifted by the surface effects, e.g. the potential of atomic step edge [21].

A powerful incentive for further extension of the realm of Kondo physics has been offered in 1988, when the idea of underbarrier tunneling in presence of Kondo center was extended on the tunneling between metallic electrodes and nanoobjects like quantum dots or small metallic grains [22, 23]. Such nanoobject may serve as a Kondo center (a localized moment) provided (i) the electron spectrum is discrete due to spatial quantization, so that the level spacing $\delta \epsilon$ exceeds the tunneling rate, (ii) Coulomb blockade prevents charge fluctuations and (iii) the electron occupation number is odd, so that the effective spin of the nanoobject is $1 / 2$. In this case the tunneling Hamiltonian may be mapped on the effective spin Hamiltonian (1), and the Kondo-like singularity arises as a zero-bias anomaly (ZBA) in tunnel transparency. This theoretical prediction was confirmed ten years later [24, 25, 26] in the experiments on planar quantum dots. Many experimental and theoretical studies then followed this experimental breakthrough ever since.

In the course of developing this new realm of condensed matter physics, further possibilities of extending the Kondo physics were subsequently discovered and exploited. Original idea of using the charge fluctuations as a source of Kondo tunneling was proposed [27] in the interim between the theoretical prediction of Kondo tunneling and its experimental verification. It was shown in this paper that in a situation, where two charge states of the quantum dot with occupation $N, N+1$ are nearly degenerate, this dot behaves as a two-level system, where the fluctuating charge configuration plays part of pseudospin, whereas the real spin projections may be treated as channel indices. Later on this idea was further developed and modified [28, 29].

Another facet of Kondo physics in nanoobjects was unveiled, when the possibility of Kondo effect in quantum dots with even electron occupation number was considered in several theoretical publications [30, 31, 32, 33]. In this case the quantum dot with a singlet ground state may become magnetically active due to external forces, and Kondo effect arises either at finite energy [30] or at finite magnetic field [31, 32, 33]. Later on it was recognized 
that in many cases the direct mapping of the original Kondo model into such system is impossible, because the effective symmetry of the pertinent nanoobject is neither $S U(2)$ nor $S U(2 N)$. The aim of this review is to describe various physical situations where the underlying nanoobject possesses complex (and in some sense unusual) symmetries which are characterized by non-compact Lie groups or combinations of such groups with discrete groups of finite rotations.

\section{SURPLUS SYMMETRIES}

Among the sources of surplus symmetries which enrich Kondo physics of nanoobjects one may find both discrete and continuous rotations stemming from complicated geometrical configurations of complex quantum dots, as well as those induced by external fields used in practical realizations of nanodevices. In this short review we will refrain from description of great variety of these devices, which may be found in current literature (see e.g., [34, 35, 36]). Fortunately, most of the relevant physics may be exposed in a relatively simple situation of electron tunneling through multivalley quantum dots in contact with metallic electrodes (leads).

A multivalley dot is an island with electrons confined by electrostatic potential in such a way that the spatially quantized electrons are distributed between several valleys. These valleys are coupled with each other by capacitive interaction and tunneling channels. Up to now there are several realizations of quantum dots with two and three valleys (double quantum dot, DQD, and triple quantum dot, TQD, respectively). Experimentally, the first such realizations of DQDs go back to mid 90-es [37, 38, 39]. It was pointed out that these objects can be treated as some forms of artificial molecules with each constituent dot playing the role of an artificial atom [40, 41, 42, 43].

Compared with DQD, fabrication of TQD is a much more difficult experimental task and the first experimental realizations of these nanoobjects appeared only recently. One may mention in this connection the realization of TQD with an "open" central valley [44]. The term "open" here means that the tunneling between the side dots and the adjacent leads is limited by strong Coulomb blockade (see below), whereas the central dot freely donates and accepts electrons to and from its own reservoir, so its role in the device is only to mediate indirect exchange between the two side dots. Another successful attempt to 
fabricate a TQD was recorded in response to a theoretical proposal [45] to use this geometry for realization of ratchet effect in tunneling through nanoobjects. In this realization [46] the charge fluctuations were suppressed by the Coulomb blockade mechanism in all three valleys. The feasibility of filling the TQD with 1,2 and 3 electrons by changing the gate voltages was demonstrated quite recently [47].

Theoretical studies of electronics in the TQD geometry were also inspired by possible applications in the field of quantum information [48, 49]. Investigation of the Kondo physics in TQD [50] was motivated by the experimental observation of molecular trimers $\mathrm{Cr}_{3}$ on gold sublayers [51] by means of the tunnel electron spectroscopy, which allows to observe Kondo-type ZBA in conductance. Later on, other properties of these trimers such as the two-channel Kondo effect [52, 53] and the interplay between the Kondo effect and AharonovBohm effect in tunnel spectroscopy [54] were considered theoretically.

Our main focus of interest is a theoretical modeling of a device consisting of a multivalley quantum dot, metallic electrodes and corresponding gates. The latter regulate the electron occupation of any particular valley and of the dot as a whole. These properties and others are predetermined by the geometrical position of the valleys relative to the metallic leads (source and drain) in the device. Basically, there are three types of such devices which are possible for describing electric circuits with a DQD connected with a source $s$ and drain $d$. They may be refereed as sequential, parallel and T-shape connections (Fig. 1a,b,c, respectively). The two small dots which combine to form the DQD may either be identical or may differ in their size. Besides, different gate voltages may be applied to different valleys.

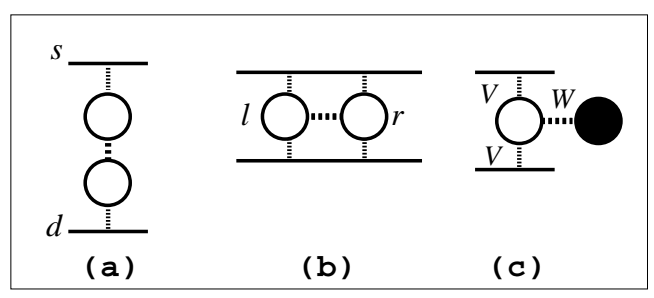

FIG. 1: Double quantum dot in sequential (a), parallel (b) and T-shape (c) geometries. Filling black marks the valley detached from the leads.

In the most symmetrical configuration of identical dots the only additional symmetry which characterizes a DQD is the permutation symmetry $P_{2}$. In close analogy with Kondo effect in magnetically doped metals, one should consider the total symmetry of a device 
'source + DQD + drain'. Then the symmetry group of the sequential configuration (Fig. 1a) contains the only discrete element, namely the $s$ - $d$ reflection axis, and this element adds nothing to the $P_{2}$ symmetry of the isolated DQD. The same statement is valid for the Tshape geometry (Fig. 1 k). In the parallel geometry the system as a whole possesses two reflection axes, namely source-drain $s$ - $d$ and left-right $l-r$, where the indices $l$ and $r$ label two valleys of DQD.

Triple quantum dots (TQD) present theoreticians (and experimentalists as well) with a richer variety of geometrical configurations and possess more symmetries (Fig. 22). Similarly to DQD these trimers may be oriented both in sequential (vertical) and parallel (lateral) geometries (Fig. $2 \mathrm{a}$ and $2 \mathrm{~b}$, respectively). The natural generalization of the T-shape geometry presented in Fig. 1c is the cross geometry (Fig. 2k). Besides, TQD may be organized in a form of a triangle, which may form a closed or open element in an electronic circuit (Figs. 2 $\mathrm{d}$,e and 2f, respectively). In the two latter cases one deals with a three-terminal tunnel device. We will call the conformations shown in Figs. 2e and 2f "ring" and "fork" configurations.

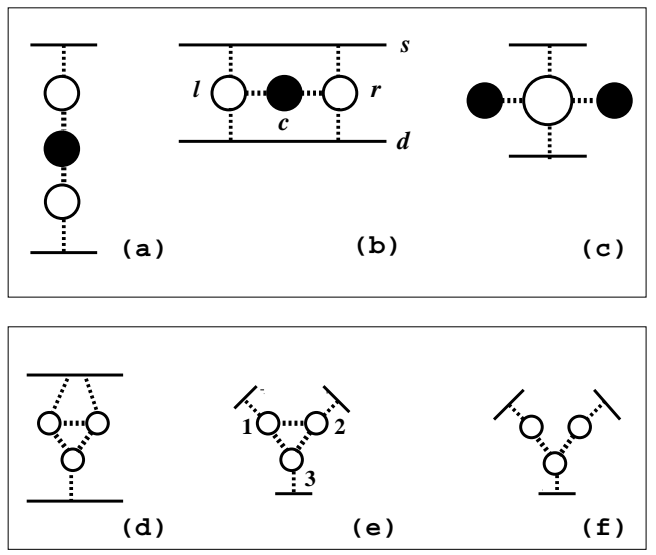

FIG. 2: Triple quantum dot in sequential (a), parallel (b), cross-shape (c), two-terminal triangular (d), three-terminal triangular (e) and fork (f) geometries.

Now let us discuss the discrete symmetry elements characteristic for the above TQD configurations. If all three valleys are equivalent, the discrete symmetry of an isolated linear TQD is that of the permutation group $P_{3}$. The contact with the leads adds one more symmetry element, the $s-d$ reflection, provided all three dots are coupled with the leads. We however consider the devices, where the central dot (filled black) is not coupled directly with the leads. Then the $P_{3}$ symmetry is lost. The only discrete symmetry element, namely 
$s-d$ reflection is left in a vertical geometry (Fig. 2a), whereas both $s$ - $d$ and $l-r$ reflections characterize a symmetry of lateral TQD (Fig. 2b). The same is valid for the cross geometry of Fig. 2k. The $P_{3}$ symmetry is inherent in the three-terminal configuration of Fig. 22. In this case it is better to use the classification of discrete rotation group $C_{3 v}$, which is isomorphic to the permutation group $P_{3}$. One may say [54, 55] that in the perfect triangular configuration the TQD imposes its $C_{3 v}$ symmetry on the device as a whole in close analogy with the Coqblin-Schrieffer-Cornut version [11, 12] of the conventional Kondo problem. In the geometries of Fig. $2 \mathrm{~d}, \mathrm{f}$ the only element which is remained of the original symmetry of triangle is the $l-r$ symmetry like in the cross geometry. External magnetic field applied perpendicularly to the plane of the triangle lowers the symmetry of a device by adding one more element, that is, chirality of the electron tunneling from the source to the drain.

Before considering the consequences of these symmetries for the Kondo physics, several introductory remarks about the derivation of the Kondo Hamiltonian are in order. In an early period of the theoretical studies, the problem of electron tunneling through short chains of quantum dots under strong Coulomb blockade restrictions was formulated in terms of the Mott-Hubbard picture [56, 57]. The theory was based on the idea that electrons injected from the source do not lose coherence when propagating through the sequence of quantum dots until they leave the chain for the drain electrode. This approach is valid only for short enough "Hubbard chain", where the tunneling $W$ between the adjacent valleys exceeds the tunneling $V$ between the dot and the metallic leads. Using generalized Landauer method, one describes the tunnel transparency in terms of the Green functions of a nanoobject in contact with the leads [58]. Such a procedure starts with diagonalization of the Hamiltonian of nanoobject with subsequent calculation of renormalization of the spectrum of quantum dot due to tunnel contact with the leads. Early studies of these problems concentrated on the calculations of the Coulomb blockade peaks which arise with changing the occupation of the valleys (so called Coulomb staircases). In terms of the Hubbard model, the Coulomb resonances are the Hubbard "minibands" [56], which arise as a result of collective Coulomb blockade [59] (Hubbard repulsion). It is known, however, that the spectral function of the Hubbard model contains also the central peak of predominantly spin origin. This peak is responsible for zero-bias anomalies in the tunnel conductance, which are at the center of our attention.

If the "chain" contains a single dot, one deals with the conventional mapping of the 
Anderson-like tunneling problem onto the Kondo-like cotunneling problem [22, 23], so that the central peak is indeed the famous Abrikosov-Suhl resonance pinned to the Fermi level of the band electrons [3, 4, 60], which is responsible for the ZBA in tunnel conductance. DQD in sequential geometry is the first non-trivial generalization of the single dot case, and one of the primary tasks is to look whether some qualitative differences from the conventional Kondo effect arise because of combining the features of Anderson and Hubbard models in the effective tunneling Hamiltonian.

In any case, working in the above paradigm [56, 57], one should derive the effective exchange Hamiltonian $H_{\text {ex }}$ in accordance with the following procedure. The starting Hamiltonian is chosen in the same form as the original Anderson impurity Hamiltonian [9]:

$$
H=H_{\text {band }}+H_{\text {dot }}+H_{\mathrm{t}}
$$

where $H_{\text {dot }}$ describes the properties of a chain detached from the lead in terms of its eigenstates $|\Lambda\rangle$ :

$$
H_{\mathrm{dot}}=\sum_{\Lambda} E_{\Lambda}|\Lambda\rangle\langle\Lambda|+Q(\hat{N}-\mathcal{N})
$$

Here $\hat{N}$ is the operator of total electron number in the dot. The last term in (3) describes the Coulomb blockade mechanism: the total occupation of the $\operatorname{dot} \mathcal{N}$ in neutral and charged states is fixed by the Coulomb blockade parameter $Q$ entering the capacitive energy of a complex quantum dot as a whole. The eigenvalues $E_{\Lambda}$ are found at fixed occupation $\mathcal{N}$ of DQD or TQD with all tunneling matrix elements $W$ and interdot capacitive interactions $Q^{\prime}$ taken into account. The tunneling Hamiltonian $H_{\mathrm{t}}$ intermixes the states from adjacent charge sectors $\mathcal{N}, \mathcal{N}^{\prime}$ due to injection of extraction of an electron from the complex dot:

$$
H_{\mathrm{t}}=\sum_{\Lambda \Lambda^{\prime}} \sum_{b=s, d} \sum_{k \sigma} V_{b k \sigma}^{\Lambda \Lambda^{\prime}} c_{b k \sigma}^{\dagger} X^{\Lambda \Lambda^{\prime}}+\text { H.c. }
$$

where $X^{\Lambda \Lambda^{\prime}}=|\Lambda\rangle\left\langle\Lambda^{\prime}\right|$ are the universal Hubbard operators [61]. The tunnel parameters $V_{b k \sigma}^{\Lambda \Lambda^{\prime}}$ are usually approximated by a single parameter $V$. In the Hamiltonian (4) these configuration changing operators describe transitions between the states belonging to different charge sectors (one of this sectors corresponds to the neutral CQD and another belong to positively or negatively charged CQD. The index $b$ enumerates the leads $(b=s, d$ in the two-terminal configurations) 
The Hamiltonian $H_{\text {band }}$ has the standard form

$$
H_{\mathrm{band}}=\sum_{b k \sigma} \varepsilon_{k} c_{b k \sigma}^{\dagger} c_{b k \sigma} .
$$

Comparing to the corresponding term in (1), this Hamiltonian contains one more index $b$. In the geometries with $s$ - $d$ reflection symmetry one may rotate the frame in such a way that the band operators are classified as even and odd operators relative to this reflection

$$
c_{e k \sigma}=2^{-1 / 2}\left(c_{s k \sigma}+c_{d k \sigma}\right), \quad c_{o k \sigma}=2^{-1 / 2}\left(c_{s k \sigma}-c_{d k \sigma}\right)
$$

In case of single and double quantum dots this rotation usually excludes the odd combination from the tunneling Hamiltonian [22]. However in case of TQD it is not necessarily the case. We will return to this question in section IV.

The cotunneling (exchange) Hamiltonian is usually obtained from (2) by means of the Schrieffer-Wolff (SW) canonical transformation [7], which excludes the states $|\Lambda\rangle$ belonging to the charge sectors $\mathcal{N} \pm 1$ from the effective Fock space. At fixed $\mathcal{N}$ we are left solely with spin degrees of freedom. In a conventional situation, the relevant symmetry is $S U(2)$ and the SW procedure results in an effective Hamiltonian (11). In case of even $\mathcal{N}$ and geometries including discrete rotations, the situation is more complicated, and the SW procedure intermixes the states $|\Lambda\rangle$ belonging to different irreducible representations of the Hamiltonian (3). The corresponding terms in the effective Hamiltonians may be expressed by means of the corresponding Hubbard operators $X^{\Lambda \Lambda^{\prime}}$. In many cases, combinations of these operators form closed algebras which generate non-compact groups $S O(N)$ or $S U(N)$ with $N>2$, describing the dynamical symmetry of complex quantum dots. Involvement of these dynamical symmetries turns the procedure of mapping the tunnel problem onto an effective exchange problem to be more complicated than in the simpler situations which were briefly described in Section I. New features of the Kondo effect arising as a result of this procedure were described for the first time using the T-shaped DQD as an example [43]. Various manifestations of dynamical symmetries in physical problems are described in the monographs [62, 63]. Some mathematical aspects of the dynamical symmetries as applied to the Kondo problem may be found in the recent reviews [64, 65]. 


\section{KONDO PHYSICS FOR SHORT CHAINS}

Short chains represented in Figs. 1 $\mathrm{a}-\mathrm{c}$, 2a-c are the most elementary objects, where many aspects of Kondo tunneling beyond the original paradigm [5, 22] of Kondo mapping may be demonstrated. There is much in common between the linear DQD and TQD in vertical and lateral geometries, although there are some effects specific only form T-shaped and cross-shaped configurations (Figs. 1 $k$ and 2k), which will also be emphasized in this section.

\section{Double quantum dots}

We start a more detailed discussion of Kondo tunneling through artificial molecules with the case of DQD in the vertical geometry (Fig. 19). Historically, this is the first generalization of a single dot problem (see above). At present, the tunneling through this simplest DQD is well understood. Following an extensive theoretical discussion of Kondo physics in vertical QD geometry [66, 67, 68, 69, 70, 71, 72, 73], it turned out that the most general description of Kondo tunneling through vertical DQD may be given in terms of $S U(4)$ and $S O(4)$ symmetries of a low-energy multiplets in cases of odd and even electron occupation $\mathcal{N}$, respectively.

As was mentioned above, the microscopic description of Kondo tunneling is analyzed in a framework of the generalized Anderson Hamiltonian (2). All generic features of Kondo mapping are seen already in the most elementary cases of $\mathcal{N}=1,2$. DQD in these charge sectors can be treated as an artificial analog of the molecular ion $\mathrm{H}_{2}^{+}$and the neutral molecule $\mathrm{H}_{2}$, respectively.

In case of $\mathcal{N}=1$ the eigenstates of the Hamiltonian $H_{\text {dot }}(\underline{3})$ are

$$
E_{1,2}=\epsilon_{d} \mp W
$$

where $\epsilon_{d}$ is the discrete level position in the isolated valley of the DQD and $W$ is the intervalley tunneling. These two levels forming the "Hubbard miniband" in the $\mathcal{N}=1$ charge sector correspond to even and odd combinations of the electron wave functions in the doublewell confinement potential of the DQD. We are interested here not in the resonance tunneling through these levels, but in the ZBA connected with the Kondo effect. The characteristic

energy $T_{K}$ which scales the Kondo effect, should be compared with the level splitting $W$. However $T_{K}$ itself depends on the level splitting, so that the comparison procedure should be 
performed self-consistently. In the limiting case $T_{K}(W) \ll W$, one may ignore the odd state $E_{2}$. Since the odd combination of the lead electron states in (6) is also excluded from the problem, we immediately see that in this case the mapping procedure (SW transformation) reduces the tunneling problem to the case of single quantum dot with the Hamiltonian (11), where the exchange constant $J$ is estimated as $J=V^{2} / E_{C}$, and $E_{C}$ is expressed via addition and extraction energies, i.e. the energy costs to add or subtract an electron on/from the quantum dot:

$$
\begin{aligned}
& E_{C}^{-1}=E_{+}^{-1}+E_{-}^{-1} \\
& E^{+}=\epsilon_{d}+Q-\epsilon_{F}, \quad E^{-}=\epsilon_{F}-\epsilon_{d} .
\end{aligned}
$$

The Kondo temperature is given by the standard equation $T_{K}=D \exp (-1 / 2 \rho J)$, where $D$ is the characteristic energy scale for the band electrons in the leads and $\rho$ is the density of states at the Fermi level $\epsilon_{F}$.

In the opposite limit $T_{K}(W) \gg W$, one may neglect $W$ when calculating $T_{K}$, so that the dot level acquires "orbital" degeneracy. Due to this quasi degeneracy the effective exchange acquires an additional factor 2 , so that the Kondo temperature in this case is $T_{K}=$ $D \exp (-1 / 4 \rho J)$. If fact, the difference between the two limiting cases is the manifestation of the $S U(4)$ symmetry, which characterizes the spin state of an electron in the double well potential. This symmetry will be described more strictly when we will turn to the case of lateral DQD. One should note that here we encountered the first manifestation of the complicated structure of spin multiplets in complex quantum dots, namely with nonuniversality of $T_{K}$ : it crucially depends not only on the parameters of the Hamiltonian but also on the effective symmetry of the low-lying spin states involved in Kondo cotunneling. Interpolation between two limiting cases may be described in terms of gradual $S U(2) \rightarrow$ $S U(4)$ crossover.

The study of the charge sector $\mathcal{N}=2$ uncovers another important aspect of the Kondo mapping procedure. This sector corresponds to the half-filled Hubbard chain, where the single-electron tunneling is suppressed by Coulomb blockade (the interior of the Coulomb diamond in terms of the theory of single-electron tunneling [34, 35]). On the surface of it, the Kondo tunneling is also not achievable because the ground state of the DQD with $\mathcal{N}=2$ is a spin singlet in close analogy with the case of hydrogen molecule $H_{2}$. However, the dynamical symmetry of DQD plays its part in this case as well. 
Indeed, in case of strong Coulomb blockade $\beta \equiv W / Q \ll 1$ the spectrum of isolated DQD consists of two low-lying spin states $E_{S, T}$ and two charge transfer excitons $E_{e, o}$ (even singlet and odd triplet), with

$$
\begin{aligned}
& E_{S}=2 \varepsilon-2 \beta W, \quad E_{T}=2 \varepsilon \\
& E_{o}=2 \varepsilon+Q, \quad E_{e}=2 \varepsilon+Q+2 \beta W .
\end{aligned}
$$

Only the low-energy Singlet/Triplet (S/T) pair is relevant for Kondo tunneling. Like in the case of $\mathcal{N}=1$, the triplet state $E_{T}$ is frozen provided the exchange gap $\Delta_{\text {ex }}=2 \beta W$ essentially exceeds $T_{K}$. In the opposite case $\Delta_{\text {ex }} \ll T_{K}$ the spin multiplet as a whole is involved in Kondo tunneling, and the Kondo-type ZBA may survive [69]. One may describe this phenomenon in terms of the theory of conventional two-site Kondo effect [75]. According to this theory, the antiferromagnetic intersite exchange $J_{12}$ competes with the single-dot Kondo temperature $T_{K 0}$. At small $J_{12}$ (small enough $W$ in our case) each spin is screened independently and the Kondo-type ground state may be achieved. However, there exists a critical value $J_{c}$, so that at $J_{12}>J_{c}$ the two spins are locked into a singlet state and the Kondo effect does not apply.

The relevant dynamical symmetry is the $S O(4)$ symmetry of S/T manifold ("spin rotator" [43]). Again we postpone the derivation of the effective Hamiltonian for the Kondo tunneling and the discussion of its observable manifestations for the case of lateral DQD. Here one should note that in the charge sector $\mathcal{N}=2$ the exchange gap $\Delta$ plays the same role as the charge-transfer gap $\mathrm{W}$ in case of $\mathcal{N}=1$ : the dependence $T_{K}(\Delta)$ is determined by a gradual symmetry crossover.

Now we turn to the parallel (lateral) geometry of Fig. 1b. A new element, which arises in this case is the possibility of separate channel for each dot. The new features brought by this additional quantum number were discussed in several publications [74, 76]. In case of two channels the conduction electrons retain additional "color" after rotation (6). We will enumerate the states corresponding to this color by the same indices $i, j=1,2$ as those used for the dots because they describe the odd and even states relative to the $l$-r reflection like those in the states (7), (9)).

We start once more with the charge sector $\mathcal{N}=1$, where a single electron in DQD is distributed between two wells. It is well known that the electron in a double well may be 
described by means of a pseudospin operator $\mathbf{T}$ with the following components"

$$
T^{z}=\sum_{\sigma}\left(d_{2 \sigma}^{\dagger} d_{2 \sigma}-d_{1 \sigma}^{\dagger} d_{1 \sigma}\right), \quad T^{+}=\sum_{\sigma} d_{2 \sigma}^{\dagger} d_{1 \sigma} \quad T^{-}=\sum_{\sigma} d_{1 \sigma}^{\dagger} d_{2 \sigma}
$$

This vector, together with four spin vectors $\mathbf{S}_{i j}$ with the following components,

$$
S_{i j}^{z}=\frac{1}{2}\left(d_{i \uparrow}^{\dagger} d_{j \uparrow}-d_{i \downarrow}^{\dagger} d_{j \downarrow}\right), \quad S_{i j}^{+}=d_{i \uparrow}^{\dagger} d_{j \downarrow} \quad S_{i j}^{-}=d_{i \downarrow}^{\dagger} d_{j \uparrow}
$$

form the set of 15 generators for the $S U(4)$ group.

To close the basis for the effective spin Hamiltonian, one has to introduce similar vector operators for the electrons in the leads, namely, the pseudospin operator $\mathbf{t}$ with components

$$
t^{z}=\sum_{k k^{\prime}, \sigma}\left(c_{2 k \sigma}^{\dagger} c_{2 k^{\prime} \sigma}-c_{1 k \sigma}^{\dagger} c_{1 k^{\prime} \sigma}\right), \quad t^{+}=\sum_{k k^{\prime}, \sigma} c_{2 k \sigma}^{\dagger} c_{1 k^{\prime} \sigma} \quad t^{-}=\sum_{k k^{\prime}, \sigma} c_{1 k \sigma}^{\dagger} c_{2 k^{\prime} \sigma} .
$$

and four spin operators $s_{i j}$ with components

$$
s_{i j}^{z}=\sum_{k k^{\prime}}\left(c_{i k \uparrow}^{\dagger} c_{j k^{\prime} \uparrow}-c_{i k \downarrow}^{\dagger} c_{j k^{\prime} \downarrow}\right), \quad s_{i j}^{+}=\sum_{k k^{\prime}} c_{i k \uparrow}^{\dagger} c_{j k^{\prime} \downarrow} \quad s_{i j}^{-}=\sum_{k k^{\prime}} c_{i k \downarrow}^{\dagger} c_{j k^{\prime} \uparrow} .
$$

The SW transformation carried out in terms of these operators results in the effective Hamiltonian,

$$
H_{\mathrm{eff}}=H_{\mathrm{band}}+H_{\mathrm{dot}}+2 \sum_{i j} J_{i j} \mathbf{S}_{i j} \cdot \mathbf{s}_{j i}+2 K \mathbf{T} \cdot \mathbf{t} .
$$

In the fully symmetric case which we describe here, all the effective exchange constants have the same value $J_{i j}=K \equiv J$. Then the Hamiltonian (14) may be reduced to a more compact and familiar form of exchange Hamiltonian in a fictitious magnetic field [74]

$$
H_{e f f}=H_{\mathrm{band}}+J \mathbf{S} \cdot \mathbf{s}-\widetilde{\mathbf{B}} \cdot \mathbf{s},
$$

where

$$
\mathbf{S}=\frac{1}{2} \sum_{\alpha \beta} f_{\alpha}^{\dagger} \hat{\Sigma}_{\alpha \beta} f_{\beta}, \quad \mathbf{s}=\frac{1}{2} \sum_{k k^{\prime}} \sum_{\alpha \beta} c_{\alpha}^{\dagger} \hat{\Sigma}_{\alpha \beta} c_{\beta}
$$

Spin fermion operators $f_{\alpha}$ and the matrix $\hat{\Sigma}$ involve 15 components, which are generators of the Lie algebra $s u(4)$, determined as

$$
\left\{\left(\tau^{+}, \tau^{-}, \tau^{z}, I\right) \otimes \sigma^{+}, \sigma^{-}, \sigma^{z}, I\right\}-\{I \otimes I\}
$$

where $\sigma^{\nu}\left(\tau^{\nu}\right)$ are the Pauli matrices in the spin (pseudospin) space and $I$ is the unit matrix. The fictitious magnetic field has only one non-zero component, namely the $\tau_{z} \otimes I$ component, 
and its magnitude is $W$, so that $\widetilde{\mathbf{B}} \cdot \mathbf{s}=W T^{z}$. Thus one concludes that in DQD with odd occupation the formally multichannel Kondo Hamiltonian (14) may be mapped onto the standard Hamiltonian (15) in the case of complete channel degeneracy, and the contribution of additional (permutation) degrees of freedom is described in this degenerate case by a fictitious magnetic field

In the charge sector $\mathcal{N}=2$ the permutation symmetry degenerates into trivial unit transformation. As to the spin degrees of freedom, one may try to described them by means of two spin operators $\mathbf{s}_{l}$ and $\mathbf{s}_{r}$ using the above mentioned analogy with the two-site Kondo problem [75]. However, such approach [77] should be used with some caution. One should take into account that in a situation, where both triplet and singlet two-electron states are involved in Kondo effect, these spins are non-independent because the kinematical constraint is imposed on the $\mathrm{S} / \mathrm{T}$ manifold by the Casimir operator $\mathcal{C} \neq \mathbf{s}_{l}^{2}+\mathbf{s}_{r}^{2}$. In accordance with the prescriptions of the theory of dynamical symmetries [62, 63, 64], one should construct two operators

$$
\mathbf{S}=\mathbf{s}_{l}+\mathbf{s}_{r}, \quad \mathbf{R}=\mathbf{s}_{l}-\mathbf{s}_{r}
$$

and impose on them the kinematic constraint

$$
\mathcal{C}=\mathbf{S}^{2}+\mathbf{R}^{2}=3
$$

Then the three components $\left(S^{z}, S^{+}, S^{-}\right)$of the vector $\mathbf{S}$ describe the states within the spin triplet and transitions between them, whereas the three components $\left(R^{z}, R^{+}, R^{-}\right)$of the vector $\mathbf{R}$ describe transitions between the singlet $\mathrm{S}$ and the states with spin projections $\mu=1,0,-1$ of the triplet $\mathrm{T}$. Six components of the vectors $\mathbf{S}, \mathbf{R}$ form a closed algebra

$$
\left[S_{\alpha}, S_{\beta}\right]=i e_{\alpha \beta \gamma} S_{\gamma},\left[R_{\alpha}, R_{\beta}\right]=i e_{\alpha \beta \gamma} S_{\gamma},\left[R_{\alpha}, S_{\beta}\right]=i e_{\alpha \beta \gamma} R_{\gamma}
$$

and form a set of generators of $S O(4)$ group. Here $\alpha, \beta, \gamma$ are Cartesian coordinate indices, and $e_{\alpha \beta \gamma}$ is the anti-symmetric Levi-Civita tensor. Two vector operators are orthogonal, $\mathbf{S} \cdot \mathbf{R}=0$. Under these constraints, two vectors $\mathbf{s}_{l, r}$ are rather fictitious than real spin operators. More detailed discussion of interconnections between two representations as well as the derivation of these operators by means of the Hubbard operators $X^{\Lambda \Lambda^{\prime}}$ may be found in [43]. If one tunneling channel couples this "spin rotator" with the reservoir of conduction electrons, then the dynamical group $S O(4)$ exhausts the spin degrees of freedom involved in Kondo tunneling. This scenario is realized in the T-shape geometry of Fig. 1k (see below). 
More complicated is the mapping procedure for the two-channel Anderson Hamiltonian describing the DQD shown in Fig $1 \mathrm{~b}$. In this case the discrete symmetry is explicitly involved in the cotunneling process, so that the $\mathrm{SW}$ transformation give the exchange part of $H_{\text {eff }}$ in the form

$$
H_{\mathrm{ex}}=2 \sum_{i j} J_{i j} \mathbf{S}_{i j} \cdot \mathbf{s}_{j i}+2 \sum_{i j} \tilde{J}_{i j} \mathbf{R}_{i j} \cdot \mathbf{s}_{j i}+2 K \mathbf{T} \cdot \mathbf{t} .
$$

DQD with l-r reflection axis, where all the states are classified as even or odd states relative to this reflection, is described by this Hamiltonian may be qualified as a "double spin rotator" [78]. The corresponding dynamical symmetry group is $P_{2} \times S O(4) \times S O(4)$

Before turning to the physical aspects of Kondo tunneling through the objects of $S U(4)$ and $S O(4)$ symmetries, one should mention that the DQD in lateral geometry with two channels is described by the same basic two-level Anderson Hamiltonian as the single planar quantum dot with two levels, one of which is occupied and another is empty. The latter model was considered in many publications [31, 32, 33, 76, 77, 79, 80, 81] (see also the review papers $[74,82])$. So the physical manifestations of "variable" symmetry are common for both systems. Fig. 3 illustrates the variation of $T_{K}$ as a function of control parameter (the tunnel splitting $W$ in case of $\mathcal{N}=1$ and the exchange gap $\Delta_{\text {ex }}$ in case of $\mathcal{N}=2$ ). In both cases the maximum value of $T_{K}$ correspond to the degeneracy points.

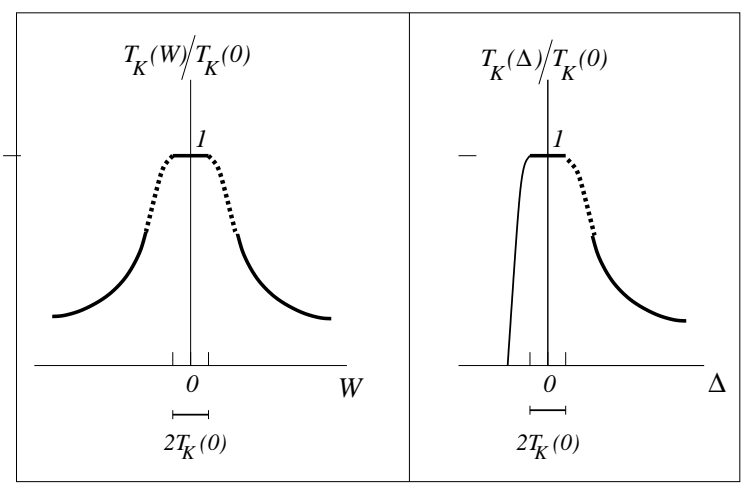

FIG. 3: Dependence of the Kondo temperature on the control parameter $W$ and $\Delta=-\Delta_{\text {ex }}$ for odd occupation (left panel) and even occupation (right panel).

This behavior may be easily understood within a perturbative (high-temperature) renormalization group $(\mathrm{RG})$ approach. This approach is based on the study of flow diagrams describing the evolution of effective coupling parameters $J_{a}(\eta)$ due to reduction of the effective energy scale $D$ of conduction electron kinetic energy [83] (label $a$ enumerates the 
vertices in the exchange Hamiltonian, $\eta=\ln D$ is the scaling variable). The general form of the system of scaling equations is

$$
\frac{d j_{a}}{d \eta}=-\sum_{b} c_{a b} j_{a} j_{b}
$$

Here $j_{a}=\rho J_{a}$ are dimensionless coupling constants and $c_{a b}$ are numerical coefficients. These equations should be solved under the boundary conditions $j\left(D_{0}\right)=\rho J_{a 0}$, where $D_{0}$ and $J_{a 0}$ are the initial conduction bandwidth and the bare exchange integrals entering the Hamiltonian $H_{\mathrm{ex}}$. When $D \sim D_{0} \gg\left(W, \Delta_{\mathrm{ex}}\right)$, one may neglect the splitting of energy terms in the system (21), and all coupling constants $J_{a}$ evolve together. When the scale $D$ is reduced down to the splitting energy $W$ or $\Delta_{\mathrm{ex}}$, the coupling parameters related to the upper level in the multiplets (7) or (9) stop to evolve, because the corresponding degrees of freedom are quenched below these energies. These parameters are $K$ both in the Hamiltonians (14) and (20), and the exchange vertices $\tilde{J}_{i j}$ related to the singlet-triplet transitions in (20). In a degenerate model (15), $T_{K}$ is partially suppressed by the fictitious magnetic field (B). As a result the temperature $T_{K}$ obtained as a solution of these equations becomes explicit function of the splitting energy. Its behavior is illustrated in the left and right panels of Fig. 3 for the charge sectors $\mathcal{N}=1,2$, respectively. In case of odd occupation $\mathcal{N}=1$ the point $W=0$ corresponds to two equivalent dots decoupled from each other. The system remains quasi degenerate till $W<T_{K}(0)=D \exp 4 / \rho J_{0}$. With further increase of the level splitting, the contribution of the state $E_{2}$ in Eq. (7) to the Kondo tunneling diminishes. In the asymptotic regime $|W| / T_{K}(0) \gg 1$, the evolution of $T_{K}$ is described by the asymptotic equation [33, 74]

$$
T_{K}(W)=\frac{T_{K}(0)^{\gamma+1}}{|W|^{\gamma}}
$$

This asymptotic curves are shown bold Fig.3. The exponent depends on the model parameter and detailed geometry of quantum dot [33, 74, 77, 78].

Similar asymptotic curve describes also the evolution of $T_{K}$ for even occupation $\mathcal{N}=2$ with $|W|$ substituted for $\Delta_{\text {ex }}$. But in this case such behavior is characteristic only for $\Delta_{\text {ex }}<0$, where the ground state is spin triplet. $T_{K}$ is maximal in the "critical point" of $\mathrm{S} / \mathrm{T}$ crossover. On the triplet side of $\mathrm{S} / \mathrm{T}$ transition it diminishes due to gradual quenching of triplet-singlet excitations described by the operator $\mathbf{R}$ in the Hamiltonian (20). On the singlet side of $\mathrm{S} / \mathrm{T}$ crossover, $\Delta_{\mathrm{ex}}>0$ the Kondo temperature falls down steeply. The singlet-triplet crossover driven by some external parameter (magnetic field, gate voltage 
etc) in DQD with even occupation is the most salient effect predicted and observed in these systems.

Additional information unveiling specific features of Kondo screening in complex quantum dots can be extracted from the temperature and magnetic field dependence of tunnel conductance $G_{\max }$ at given $W$ or $\Delta_{\text {ex }}$. Its behavior was discussed time and again [71, 76, 77, 81, 82] The most interesting is the behavior of conductance in the charge sector $\mathcal{N}=2$.

On the triplet side of $\mathrm{S} / \mathrm{T}$ crossover $G_{\max }(T)$ increases with decreasing $\mathrm{T}$. At high $T \gg$ $T_{K}\left(\left|\Delta_{\text {ex }}\right|\right)$, it grows logarithmically,

$$
G_{\max }(T) / G_{0} \sim \ln ^{-2}\left(T / T_{K}\right)
$$

where $G_{0}=2 e^{2} / h$ is the limiting value of tunnel conductance in a Kondo regime (unitarity limit, where the sum of all phase shifts on the Fermi levels $\delta_{i \sigma}$ equals $\pi / 2$ ). This limiting value quantify the tunnel conductance at $T-0$. According to the Friedel sum rule [8, 60], generalized for the two-channel geometry [79, 80] one has

$$
G(T=0)=G_{0} \sin ^{2}\left[\frac{\pi}{2}\left(\bar{n}_{2}-\bar{n}_{1}\right)\right]
$$

where $\bar{n}_{(2,1)}$ is the change in number of electrons under the Fermi level due to Kondo screening in two channels (even and odd). In terms of the phase shifts the argument of the sine function in the r.h.s. of Eq. (24) reads $\frac{1}{2} \sum_{i \sigma} \delta_{i \sigma}$. This means that the demand of spin rotation invariance and $l-r$ symmetry means that the phase shift in each channel reaches $\pi / 4$ at $T=0$. Similarly to the theory of Kondo scattering [16], the deviation from the unitarity limit is of standard Fermi-liquid character

$$
G(T) / G_{0}=\left[1-\pi\left(T / T_{K}\right)^{2}\right]
$$

at $T \ll T_{K}$. The monotonous interpolation [82] between two limiting temperature regimes (23) and (25) may be violated due to multistage Kondo effect. We will discuss this regime, when considering the case of triple quantum dots (see below).

The influence of external magnetic field $B$ on the tunnel conductance at low $T \ll B$ is easily tractable [82]: in accordance with the general theory of Kondo effect in presence of Zeeman splitting of the levels in the dot, in this regime $T$ should be substituted for $B$ in the asymptotic equations (23) and (25). At low $T \ll T_{K}$ additional information may be obtained by means of the NRG method [81]. Due to the loss of spin rotation symmetry the 
phase shifts $\delta_{i \sigma}$ become explicit functions of magnetic field. In a single channel case this dependence is scaled by $T_{K}$, namely $\delta_{\sigma}(B)=\delta(0)+\sigma\left(B / T_{K}\right)$. In a two-channel regime, level splitting $W$ between the dot levels enters this dependence explicitly. A simple equation describing this dependence was derived analytically [80],

$$
\delta_{i \sigma}=\delta(0)+\sigma\left(B / T_{i}\right)+(-1)^{i}\left(B / T_{i}^{\prime}\right)+O\left(B^{3}\right)
$$

Here the parameters $T_{i}$ and $T_{i}^{\prime}$, which scale the field $B$ depend on $W$. NRG calculations [81] show great variety of magnitoconductance curves $G(B, W)$.

On the singlet side of $\mathrm{S} / \mathrm{T}$ crossover the ground state of DQD is nonmagnetic, so that there is no room form Kondo-type ZBA at $T=0$ (see the right panel of Fig. 3). However, at $T>\Delta_{\text {ex }}$ the triplet state is still involved in perturbative scaling equations (21), so that $G(T)$ grows with decreasing $T$ in accordance with Eq. (23). The temperature T/S crossover occurs at $T \sim \Delta_{\mathrm{ex}}$, and conductance starts to fall with further decrease of $T$ ending with exponentially small value of $G(T=0)$ [76]. Similar effect should be observed in the behavior of $G$ as a function of source-drain voltage $v_{d s}$. At $e v_{d s}>\Delta_{\text {ex }}$ the DQD shows Kondo tunneling, but with lowering bias the triplet channel is quenched, and conductance shows up a zero field dip instead of zero field peak. Apparently this type of crossover was observed [84] in a two-orbital planar dot with even occupation.

\section{T-shape geometry}

In a T-shape geometry one of two dots is detached from the leads (Fig. 11c). In the first experimental device of this type [38], the role of the side (right) dot was to control single-electron tunneling through the left dot. Recently several new effects related to the Kondo-regime were discovered in this geometry.

First, it was shown [43] that the T-shape double dot with even occupation $\mathcal{N}=2$ demonstrates the properties of spin rotator with $S O(4)$ symmetry. The simplest form of the Hamiltonian (20), namely

$$
H_{\mathrm{ex}}=2 J \mathbf{S} \cdot \mathbf{s}+2 \tilde{J} \mathbf{R} \cdot \mathbf{s}
$$

was derived just for this model. Besides, it was shown that in the asymmetric T-shape DQD, where the Coulomb blockade in the right dot is sufficiently larger than in the left dot, the $\mathrm{S} / \mathrm{T}$ crossover may occur because of the many-body logarithmic renormalization [85, 86], 
which is determined by the renormalization group invariants $E_{\Lambda}^{*}$, namely

$$
E_{\Lambda}^{*}=E_{\Lambda}(D)-\pi^{-1} \Gamma_{\Lambda} \ln \left(\pi D / \Gamma_{\Lambda}\right)
$$

The level crossing is possible because the inequality $\Gamma_{T}>\Gamma_{S}$ for the tunneling rates $\Gamma_{\Lambda}$ is realized for asymmetric DQD, so that the renormalization of the triplet level $E_{T}$ is stronger than that of the singlet state $E_{S}$. Similar effect may be achieved in a symmetric DQD by means of the gate voltage applied to the side dot (later on the possibility of inducing the $\mathrm{S} / \mathrm{T}$ crossover by means of the the gate voltage was found also for two-orbital planar dots [87]).

Another interesting possibility arises at the odd occupation $\mathcal{N}=1$, where the strong Coulomb blockade exists only in the side dot [88, 89, 90, 91]. It was found that in this case the Kondo resonance arises on the background of otherwise non-correlated transport between the electrodes via the right dot. The interference between the resonance scattering and free propagation is known as Fano effect discovered originally in the optical absorption spectra of free atoms [92]. The Fano effect in atoms is described by the same Hamiltonian as the Anderson impurity hybridization [9], so there is no wonder that similar effect was found in the resonance impurity scattering in metals [93]. Since the single electron transport through the quantum dot is also described by the Anderson Hamiltonian (2), the Fano effect ubiquitous in resonance scattering was expected in tunnel conductance of quantum dots, and the structures of characteristic Fano-type form were indeed observed in the tunneling spectra of planar quantum dot [94]. In the latter case the Fano effect arises due to the interference between the resonance level in the quantum dot and the band continuum in the leads. The corresponding contribution to the conductance has the form

$$
G_{\text {Fano }}(\epsilon)=G_{0} \frac{(\varepsilon+q)^{2}}{\varepsilon^{2}+1}
$$

where $\left.\varepsilon=2\left(\epsilon-\epsilon_{d}\right)\right) / \Gamma, q$ is the so called asymmetry parameter predetermined by the spectral characteristics of the lead electrons, $\Gamma=\pi \rho V^{2}$ is the tunneling rate. In simple terms, Fano effect is nothing but modification of the spectral density of conduction electrons due to its repulsion from the resonance level superimposed on the continuous spectrum.

In a T-shape geometry the Fano effect arises due to superposition of the Abrikosov-Suhl resonance created by the Kondo effect in the right dot on the continuous tunneling spectra of the system 'source - left dot - drain'. As was noticed by Kang et al [88], this Fano-Kondo 
effect looks as an antiresonance in conductance: instead of the standard Friedel-Langer formula (24), one has

$$
G(T=0)=G_{0} \cos ^{2}\left[\frac{\pi}{2} \bar{n}_{r}\right]
$$

where $n_{r}$ stands for the average occupation number of the right dot. Such "unitarity limit" for conductance means that in the T-shape geometry, the Kondo effect in the right dot results in complete suppression of resonance tunneling through the left dot and a dip arises in the tunnel conductance instead of the usual peak. As was mentioned above, the phase shifts in the Kondo regime are spin dependent, so the modification of tunnel spectra due to destructive Kondo-Fano interference is also spin-dependent, and the T-shape dot in this regime may work as a spin filter in external magnetic field [95].

One more advantage of the TQD geometry is the possibility to approach the two-channel Kondo effect [96, 97]. Such possibility arises in the geometry of Fig. 11c, when the Coulomb blockade is strong in the left dot and the right dot is big enough so that the level spacing is less than the tunnel rate, but the Coulomb blockade is still strong and fixes the electron occupation number. In such conditions the way opens to overcome the main difficulty in realizing a physical system that materializes the two channel Kondo model. The necessary precondition for this regime is in creating two separate channels that equally screen the spin [15]. In conventional setups an electron from one channel that hops on the dot may hop to the other channel and thus cause mixing between the channels. This mixing lead eventually to two "eigen channels" with one channel coupled stronger than the other one. The channel with the stronger coupling fully screen the spin and the other channel is decoupled, and we thus have again the single channel Kondo case. It was suggested [96] to overcome this mixing problem by using a large quantum dot as an additional channel. Then, the free leads form one channel [even one in accordance with our classification (6)] and the large dot forms the second channel. The channels do not mix as transfer of electrons between them charges the large dot. As a result, the SW transformation maps the original Anderson Hamiltonian for a T-shape quantum dot onto the two-channel exchange Hamiltonian

$$
H_{2 \mathrm{ch}}=\sum_{\gamma, k} \epsilon_{\gamma, k} c_{\gamma, k}^{\dagger} c_{\gamma, k}+\Sigma_{\gamma} J_{\gamma} \mathbf{S} \cdot \mathbf{s}_{\gamma}+B S^{z}
$$

Here the channel index $\gamma$ stands for the even lead-dot channel and the states in the large dot $r$. The tunnel conductance in the 2-channel non-Fermi liquid regime is realized at $J_{2}=J_{r}$. It 
demonstrates specific temperature dependence. In accordance with predictions of conformal field theory [97, 98],

$$
G_{\mathrm{nfl}}(T)=\frac{G_{0}}{2}\left(1-\sqrt{\pi T / T_{K}}\right)
$$

Practically, the zero temperature limit cannot be achieved because the Kondo screening in the large dot is quenched due to discreteness of its electron spectrum at $T \sim \delta \epsilon$.

Unlike the single channel case, the magnetic field is a relevant parameter in the two channel case [99]. Introducing the channel anisotropy parameter $\Delta_{\mathrm{ch}}=J_{r}-J_{2} \ll J_{2}$, which describes deviation from the 2-channel fixed point $\left(\Delta_{\mathrm{ch}}=0\right)$, the following equation for the magnitoconductance may be derived [97, 99]

$$
G_{\mathrm{nfl}}(T, B)=G_{0}\left[\frac{1}{2}+a \operatorname{sign}\left(\Delta_{\mathrm{ch}}\right) \frac{B_{\Delta}}{B}-b \frac{B}{T_{K}} \ln \frac{T_{K}}{B}\right]
$$

where $B_{\Delta}=\Delta_{\mathrm{ch}} T_{K} / J_{2}^{2}$, and $a, b$ are positive numerical coefficients of the order of 1

\section{Triple quantum dots}

In this section we consider new features of Kondo mapping which are characteristic for the chains consisting of three dots (Fig. $2 \mathrm{a}$ a-c). Simple increase of the number of sites in the chain promises no new qualitative results, so we will discuss here the configurations where the central dot differs from two side dots in its size (and hereby by the magnitude of the Coulomb blockade parameter $Q_{c}$ ), but the latter dots are identical, so that the TQD retains its reflection symmetries. Both conceivable situations, namely $Q_{c} \gg Q_{s}$ and $Q_{c} \ll Q_{s}$ will be considered (the indices $c, s$ are used in this section to indicate the physical quantities related to the central and side dots, respectively). In the first experimental realization of

TQD [44] the former option was chosen, whereas the first theoretical study [78] was devoted to to the second possibility.

In a TQD with "open" central dot and $Q_{c} \ll Q_{s}$, its role in formation of Kondo tunneling regime reduces to providing the channel for indirect RKKY-type mechanism of exchange between two localized spins formed in side dots. Thus, from the theoretical point of view this problem is in fact may be mapped onto that for a DQD (see Section III.A) with specific exchange mechanism. The Kondo effect may be described in terms of two-site Kondo Hamiltonian, where the trend to interdot spin coupling competes with the trend to individual Kondo coupling between two side dots and the electrons in the leads [100, 101]. One may 
mention in this connection the phase diagram of effective two-site Kondo effect calculated in a framework of the model, where the pair of spins is coupled to the linear electron chain in a side geometry [102].

Qualitatively new features of Kondo mapping problem were found out in the opposite limiting case $Q_{c} \gg Q_{s}$. As follows from the general scheme of Kondo-mapping based on the dynamical symmetry of artificial molecule (Section III.A), the form of effective exchange Hamiltonian (20) depends on the structure of spin multiplet of isolated multivalley quantum dot. Up to now only two possibilities were exploited: spin and orbital doublet for odd occupation $\mathcal{N}=1$ and single-triplet pair for even occupation $\mathcal{N}=2$. Study of TQD with odd and even occupations $\mathcal{N}=3$ and $\mathcal{N}=4$ give us new opportunities [78, 103].

To demonstrate these opportunities let us consider TQD in lateral geometry. Genesis of spin multiplets may be understood from a general set-up illustrated by Fig. 4, In case of

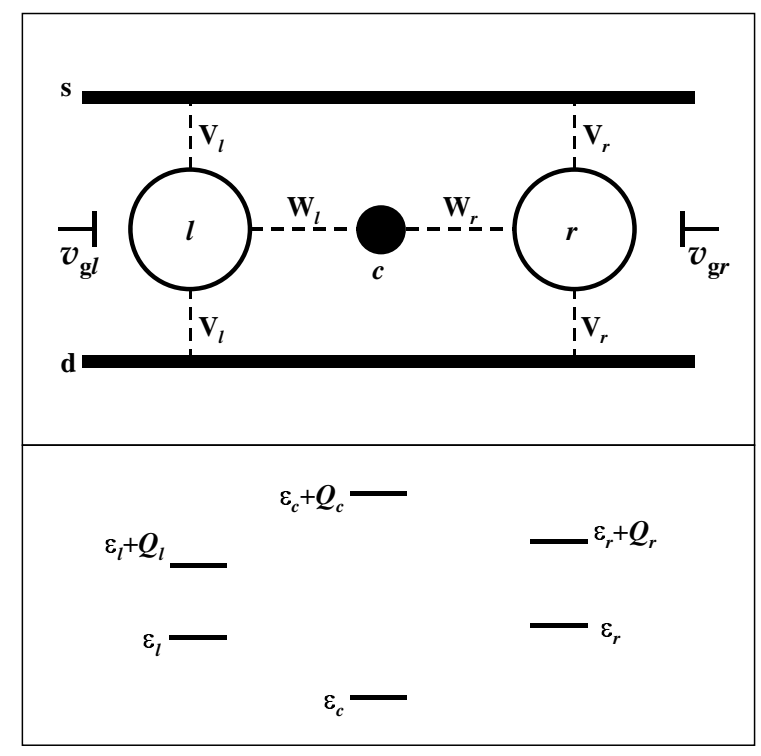

FIG. 4: TQD in parallel geometry and energy levels of each dot $\varepsilon_{a}-e v_{g a}$ at $W_{a}=0$.

$\mathcal{N}=3$ three electrons are distributed over three dots in such a way that the state with doubly occupied central dot is suppressed by strong Coulomb blockade $Q_{c}$. In accordance with the Young tableaux for a system with $l-r$ permutation symmetry, the spin multiplet consists of two doublets with $S=1 / 2$ having even and odd symmetry relative to this permutation and one quartet $S=3 / 2$ with full orbital symmetry. In case of $\mathcal{N}=4$ the spin manifold consists of two spin singlets $S_{e, o}$ and two triplets $T_{e, o}$, both even and odd relative to $l-r$ permutation. Varying the gate voltages $v_{g l}, v_{g r}$ and playing with tunnel parameters $V_{l, r}$ and $W_{l, r}$, one may 
break l-r symmetry (Fig. 4) and change the singlet-triplet splitting, so that the spin states are classified as $S_{l, r}$ and $T_{l, r}$. The relative positions of energy levels in spin multiplets evolve as a function of model parameters and various types of level crossings occur (see [78] for detailed calculations). Similar situation arises for vertical configuration of Fig. 2a.

In accordance with general theory of dynamical symmetries [64], quasi degeneracy of lowlying states in spin multiplets within the energy scale $\sim T_{K}$ generates special symmetries of TQD. For example, if the multiplet of low-lying states consists of two singlets and one triplet, the relevant dynamical symmetry is $S O(5)$. If this multiplet is formed by two triplets and one singlet, the corresponding symmetry is $S O(7)$, etc. The methods of constructing the generators for these groups are described in details in the reviews [64, 65]. As a result, an unique opportunity arises to change the value of index $n$ characterizing the symmetry $S O(n)$ of TQD by varying the gate voltages and other experimentally controllable parameters of a device. The phase diagram of vertical TQD with $\mathcal{N}=4$ calculated in [78] is presented in Fig. 5. This diagram shows great variety of phases with different symmetries from the

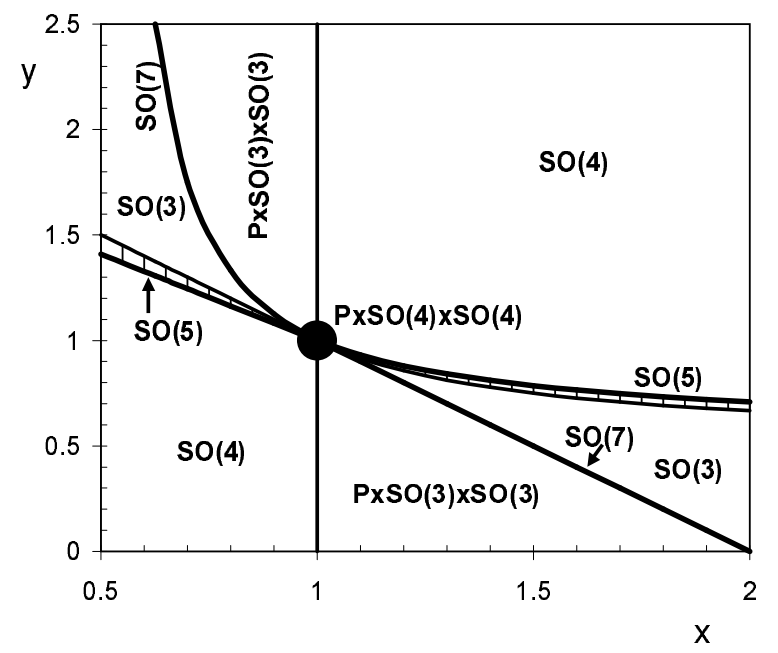

FIG. 5: Dynamic symmetries in TQD: Phase diagram in coordinates $x=\Gamma_{l} / \Gamma_{r}, y=\left(\varepsilon_{l}-\varepsilon_{c}\right) /\left(\varepsilon_{r}-\right.$ $\left.\varepsilon_{c}\right)$.

most symmetric one $P_{2} \otimes S O(4) \otimes S O(4)$ to conventional $S O(3)$ phase where the ground state of TQD is spin triplet, or non-Kondo singlet ground state (shaded areas). Each phase is characterized by its own $T_{K}$, and this means that the ZBA in conductance should follow the change of the Kondo temperature, so that each crossover from one symmetry to another is accompanied by the abrupt change of conductance at given temperature. 
At $\mathcal{N}=3$ we meet a somewhat unexpected situation where Kondo tunneling in a quantum dot with odd occupation demonstrates the exchange Hamiltonian of a quantum dot with even occupation. The reason for this scenario is the specific structure of the wave function of TQD with $N=3$. The corresponding wave functions are vector sums of states composed of a "passive" electron sitting in the central dot and singlet/triplet $(\mathrm{S} / \mathrm{T})$ two-electron states in the $l, r$ dots. Then using certain Young tableaux [78], one concludes that the spin dynamics of such TQD is represented by the spin 1 operator $\mathbf{S}$ corresponding to the $l-r$ triplet, the corresponding R-operator $\mathbf{R}$ and the spin $1 / 2$ operator $\mathbf{s}_{c}$ of a passive electron in the central well. The latter does not enter the effective Hamiltonian $H_{\mathrm{ex}}$ but influences the kinematic constraint via Casimir operator $\mathcal{C}=\mathbf{S}^{2}+\mathbf{M}^{2}+\mathbf{s}_{c}^{2}=\frac{15}{4}$. The dynamical symmetry is therefore $S O(4) \otimes S U(2)$, and only the $S O(4)$ subgroup is involved in Kondo tunneling. Similar situation, although for different reasons is realized in fork and cross geometries (see below).

Remarkable symmetry reduction occurs in external magnetic field [78]. First example of such reduction was found in a situation where the exchange splitting of $\mathrm{S} / \mathrm{T}$ multiplet (symmetry group $S O(4)$ ) is compensated by the Zeeman splitting [31], so that the up spin projection $|T 1\rangle$ of triplet forms a pseudospin with singlet $|S\rangle$ and the symmetry reduction $S O(4) \rightarrow S U(2)$ takes place. In case of TQD with $S O(5)$ symmetry, due to the same compensation the system may be left in a subspace $\left\{T 1_{l}, S_{l}, S_{r}\right\}$. The symmetry reduction in this case is $S O(5) \rightarrow S U(3)$, and the Anderson Hamiltonian is mapped on a very specific anisotropic Kondo Hamiltonian involving only operators $\mathbf{R}_{i}$,

$$
H_{\mathrm{ex}}=\sum_{i j} \sum_{\mu \nu} J_{i j}^{\mu \nu} R_{i}^{\mu} s_{j}^{\nu}
$$

where $\mu, \nu$ are cartesian components of scalar product. Here the Kondo effect is described exclusively in terms of dynamical symmetry.

Another non-standard manifestation of Kondo mapping for linear TQD is the possibility of two-channel Kondo effect in vertical geometry of Fig. $2 \mathrm{a}$ with $Q_{c} \gg Q_{s}$ at $\mathcal{N}=3$ with preserved $s$ - $d$ mirror symmetry [103]. The strong Coulomb blockade in central dot prevents direct $s$ - $d$ tunneling. The cotunneling is possible only because the wave functions of electrons centered on the side dots have small tails on the central dot. It is crucially important that the standard rotation (6) does not eliminate the odd channel from the tunneling Hamiltonian in TQD. In the situation, where the ground state of TQD is the spin doublet with even parity 
$\left|D_{e}\right\rangle$, the SW transformation for the original Anderson Hamiltonian results in anisotropic two-channel exchange Hamiltonian,

$$
H_{\mathrm{ex}}=J_{s} \mathbf{S} \cdot \mathbf{s}_{s}+J_{d} \mathbf{S} \cdot \mathbf{s}_{d}+J_{s d} \mathbf{S} \cdot\left(\mathbf{s}_{s d}+\mathbf{s}_{d s}\right)
$$

Due to the presence of nondiagonal vertex $J_{s d}$ the incurable orbital anisotropy arises: the tunneling through two channels is controlled by the parameters $J_{ \pm}=\left(J_{s}+J_{d}\right) / 2 \pm$ $\sqrt{\left.\left(J_{s}-J_{d}\right)^{2} / 4+J_{s d}^{2}\right)}$. In accordance with the theory of two-channel Kondo effect [15], this anisotropy makes the 2-channel fixed point unattainable, but due to strong Coulomb blockade in central dot $J_{s d}=\sim V^{2} W^{2} / Q_{c} \varepsilon_{d}^{2}$ is extremely small, so that one may approach the fixed point close enough, and the predecessor of 2-channel regime may be observed experimentally as a dip in conductance $G$ as a function of the difference of gate voltages $v_{g s}-v_{g d}$ applied to the side dots (this difference controls the degree of channel anisotropy).

$T Q D$ in cross and fork geometry

To complete the studies of linear artificial molecules we consider in this section the configurations shown in Fig. 2 (c,f). We have seen above that one may meet the situation, where the linear TQD with odd occupation and half-integer spin demonstrates the Kondo physics characteristic for even occupation with integer spin due to the fact that one of the electrons in the dot does not participate in tunneling. Here we will discuss two more mechanisms of such "disguise" [104].

One of these mechanisms is realized in cross geometry at occupation $\mathcal{N}=3$ under condition $Q_{s} \gg Q_{d}$ for Coulomb blockade parameters. In this case two side electrons are passive: the tunneling between source and drain occurs through the central dot. However, these passive electrons influence Kondo mapping because they are responsible for the parity of the 3 -electron wave function relative to the $l-r$ reflection. Diagonalization of the low-energy spin states shows that it consists of three spin doublets and one spin quartet, and the lowest state in this manifold is the doublet $D_{u}$, which is odd relative to the mirror reflection (see [104] for details). Although the wave functions of two passive electrons do not enter explicitly in the indirect exchange integral arising due to SW transformation, this integral changes its sign due to odd parity of the state $D_{u}$. Thus, in contrast to the standard paradigm of Kondo mapping, the effective exchange Hamiltonian corresponds to ferromagnetic coupling, which 
is irrelevant to Kondo effect, and the Kondo-type ZBA does not arise in this case in spite of the fact that the net spin of quantum dot is $1 / 2$.

However, this is not the end of the story. The excited states in the spin multiplet which are Kondo active, influence the tunnel transparency and conductance at finite temperature and finite energies of incident electron due to non-trivial dynamical symmetry of TQD described above. The states involved in the Kondo effect are the even spin doublet $D_{g}$ and the quartet $Q$, so that the overall dynamical symmetry of TQD in cross geometry is $S U(2) \otimes S U(2) \otimes S U(2)$. We meet here the situation, which reminds the "two-stage" Kondo effect in DQD with $\mathcal{N}=2$, on the singlet side of $\mathrm{S} / \mathrm{T}$ crossover (see Fig. 3b and subsequent discussion). Here, however, there are three stages of Kondo screening, where the states $Q$ and $D_{g}$ are quenched one after another with decreasing energy or temperature and the Kondo screening eventually stops at zero $T$. Besides, the hierarchy of tunneling rates $\Gamma_{Q}>\Gamma_{D g}>\Gamma_{D u}$ exists in this charge sector, so the level crossing controlled by the parameters of TQD is possible in accordance with Eq. (28). This level crossing is shown in Fig. 6. Here the scaling variable is chosen in the form $\eta=\ln \left(\pi D / \Gamma_{Q}\right)$. The value of $\bar{D}$ is determined from the crossover condition $\bar{D}_{\Lambda} \approx E_{\Lambda}\left(\bar{D}_{\Lambda}\right)$, where the renormalization (28) changes for the SW regime with fixed charge, $\bar{D}_{0}$ is the initial value of scaling variable.

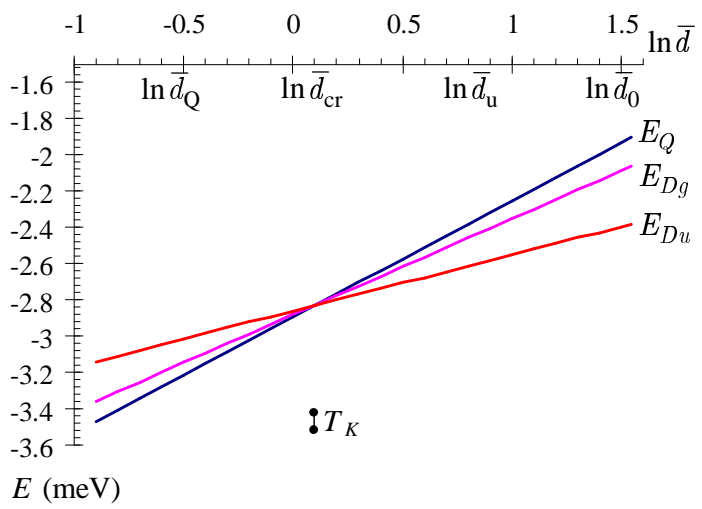

FIG. 6: Flow diagram for the levels $E_{\Lambda}$ determined by the scaling invariant (28). $\Lambda=D_{u}, D_{g}, Q$, $\bar{d}=\pi \bar{D} / \Gamma_{Q}$. Energy is measured in meV units (see text for further explanations).

Three points $\left(\ln \bar{D}_{u}, \ln \bar{D}_{Q}, \ln \bar{D}_{c r},\right)$ on the abscissa axis correspond to three values of the control parameters where the crossover to the $\mathrm{SW}$ regime occurs for the ground states $E_{D_{u}}$, $E_{Q}$ and the completely degenerate ground state, respectively. By means of appropriate variation of the control parameters, the system may be transformed from a non-Kondo 
regime with the ground state $E_{D u}$ to the underscreened Kondo regime with the ground state $E_{Q}$ and spin $S=3 / 2$. In accordance with the general theory of Kondo mapping (Section III.A), $T_{K}$ is maximum in the point of maximum degeneracy. Evolution of $T_{K}$ is shown in Fig. 7, which should be compared with Fig. 3b. Unlike the case of singlet/triplet crossover

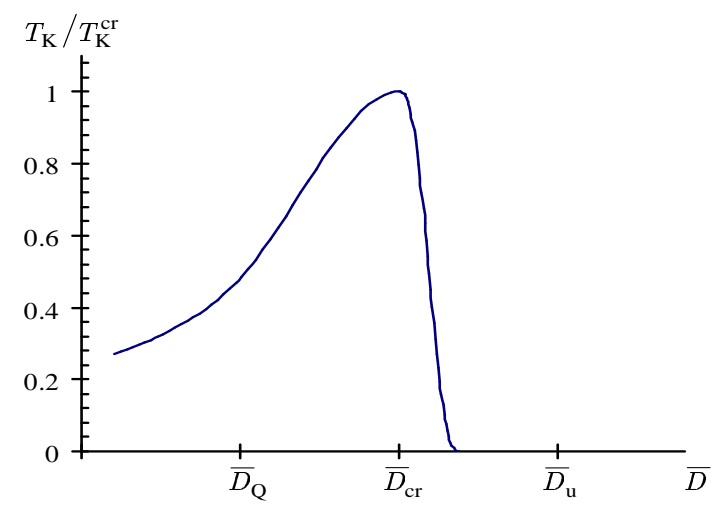

FIG. 7: Evolution of $T_{K}$ as a function of a control parameter $\bar{D}$.

in DQD with $\mathcal{N}=2$, here one deals with the crossover from the non-Kondo spin doublet $D_{u}$ to the Kondo spin quartet $Q$ via the highly degenerate region of $S U(2) \otimes S U(2) \otimes S U(2)$ symmetry.

Let us turn to the fork geometry shown in Fig. 2f. In this geometry non-trivial Kondo physics arises already in the simplest case of odd occupation $\mathcal{N}=1$ in a situation with the $l-r$ mirror symmetry. The fork may be considered as a "quantum pendulum" [49, 105]. Three one-electron eigenvalues are

$$
E_{D_{b, a}}=\epsilon_{c} \mp 2 W^{2} / \Delta, \quad E_{D_{n}}=\epsilon_{s},
$$

$\left(\Delta=\max \left\{\left|\epsilon_{s}-\epsilon_{c}\right|,\left|Q_{c}-Q_{s}\right|\right\}\right)$ The eigenstates are classified as a non-bonding spin doublet $D_{n}$ (odd combination of the wave functions centered in the sites 1,2) and bonding/antibonding pair $D_{b, a}$ of corresponding even combination with the state centered in the cite 3 (see [104] for details). The latter pair is the analog of resonant valence bonds (RVB) known in "natural" molecules. To describe this pendulum one should introduce the pseudospin vector $\mathbf{T}$ defined in Eq. (10) and work with the Hamiltonian (14). Like in the cross geometry, the level crossing effect as a function of control parameter takes place (Fig. 8).

Here $T_{K}$ is nonzero on both sides of the crossover and its evolution is described by the bell-like curve similar to that in Fig. 3a (although slightly asymmetric). However the 


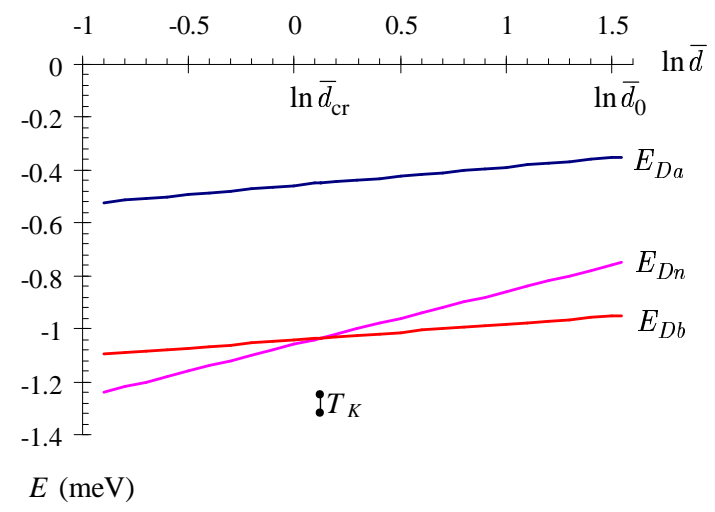

FIG. 8: Flow diagram for the levels $E_{\Lambda}$ of the TQD in fork geometry.

tunnel conductance is drastically influenced by the pendulum structure of the electron wave function. In the three-terminal fork geometry, one should consider separately the situations, where the bias voltage is applied between the leads 1 and 2 and between the leads 1 and 3 . We define the corresponding components of tunnel conductance as $G_{22}$ and $G_{33}$, respectively. The Kondo anomaly in $G_{22}$ is predetermined by the RVB pair, and the ZBA roughly follows the evolution of $T_{K}$ through the crossover. More peculiar behavior is expected in 1-3 channel because the non-bonding state $D_{n \sigma}=2^{-1 / 2}\left(d_{1 \sigma}^{\dagger}-d_{2 \sigma}^{\dagger}\right)$ is detached from the lead 3. As a result the Kondo contribution to $G_{33}$ manifests itself as a finite bias anomaly (FBA) in a situation where the ground state of TQD is $E_{D_{n}}$. Tunnel conductance as a function of bias voltage in both channels is illustrated in Fig. 9. The dip in the curve $b$ on the right panel reminds similar dip in the tunnel conductance of DQD with $\mathcal{N}=2$ on the singlet side of $\mathrm{S} / \mathrm{T}$ crossover [76, 84].

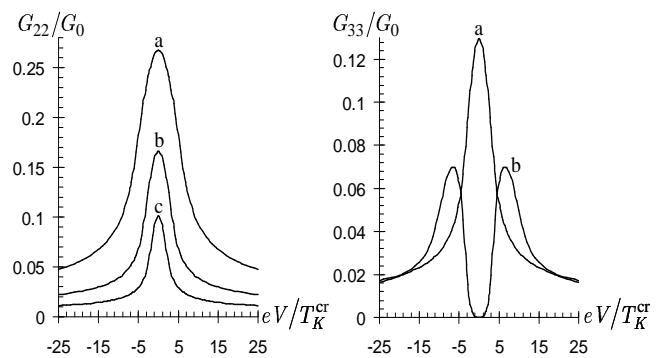

FIG. 9: Left panel: Tunnel conductance in the channel '2-1' for $\bar{D}=\bar{D}_{c r}$ (a), $\bar{D}>\bar{D}_{c r}$ (b) and $\bar{D}<\bar{D}_{c r}$ (c). Right panel: Tunnel conductance in the channel '3-1' for $\bar{D}>\bar{D}_{c r}$ (a) and $\bar{D}<\bar{D}_{c r}$ (b).

Thus, we see that some manifestations of the Kondo effect in TQD with odd occupation 
may mimic those for DQD with even occupation due to specific influence of the mirror reflection on the structure of the electron wave functions in trimers.

\section{KONDO PHYSICS FOR SMALL RINGS}

In previous sections we discussed the Kondo effect in short "Hubbard chains" in contact with metallic reservoirs. Meanwhile, the Hubbard-like objects were studied also in closed ring geometries as well (see, e.g., [106, 107]). Experimentally Kondo effect on closed rings was observed on gutter-like dots in planar geometry [108, 109], but the impact to theoretical investigation of Kondo effect in ring-like nanoobjects [50] was given by experimental studies of Co trimers adsorbed on metallic sublayer [51]. It was shown that the basic symmetry of equilateral triangular triple quantum dot (TTQD, Fig. 2e) with odd occupation is $S U(4)$ due to the interplay between the spin and orbital degrees of freedom, similar to that in two-orbital DQD (see Section III.A). Special attention was paid to the case $\mathcal{N}=3$ which models triangular Co trimer [53, 110]. In this charge sector the effective spin Hamiltonian contains not only exchange interaction between the spins in the dots and adjacent leads, but also the two-site Heisenberg exchange between spins in the neighboring dots. Magnetic frustrations in triangular geometry affect the spin state and therefore influences the Kondotype ZBA in tunneling spectra. These spectra were calculated by the numerical RG and quantum Monte-Carlo methods. Besides, it was found [53] that in case of complete channel isotropy the non-Fermi-liquid regime arises from the interplay of magnetic frustrations and Kondo physics.

Another phenomenon, which interplays with the Kondo physics is the Aharonov-Bohm oscillation of tunnel transparency in magnetic field directed perpendicularly to the plane of triangle. This effect may be seen already for the Hubbard ring with 1/3 occupation (TTQD with $\mathcal{N}=1$ ), where there is no room for exchange interaction between spins localized in neighboring sites and concomitant magnetic frustrations. The starting point for solving the problem of interplay between Kondo and Aharonov-Bohm phenomena [54, 111] is the Anderson Hamiltonian (2) rather than the exchange Hamiltonian (14).

In accordance with the general scheme discussed in Section II, one should start with the diagonalization of the Hamiltonian of 3-site Hubbard ring. The point symmetry of this equilateral triangle is $C_{3 v}$. This group describes the "orbital" degrees of freedom, whereas the 
continuous spin symmetry is usual $S U(2)$ symmetry of spin $1 / 2$. According to the irreducible representations of $C_{3 v}$ group, the spectrum of TTQD consists of three levels $\Lambda=D A, D E_{ \pm}$. Here as usual $D$ stands for spin doublet, $A$ is the fully symmetric orbital singlet and $E_{ \pm}$are two components of orbital doublet. The energies of these states in out-of-plane magnetic field $B$ are

$$
E_{D \Gamma}(p)=\epsilon-2 W \cos \left(p-\frac{\Phi}{3}\right) .
$$

such that for negative $W$ and for $B=0, p=0,2 \pi / 3,4 \pi / 3$ correspond respectively to $\Lambda=A, E_{ \pm}$with the ground state $D A$, so that the orbital degrees of freedom are quenched at low temperature and the SW mapping procedure ends with conventional Kondo Hamiltonian (11). However, using the magnetic field as a control parameter, one may initiate level crossing by varying the magnetic flux $\Phi$ through TTQD. This level crossing is shown on the upper panel of Fig. 10, In each crossing points and its nearest vicinity the dynamical symmetry

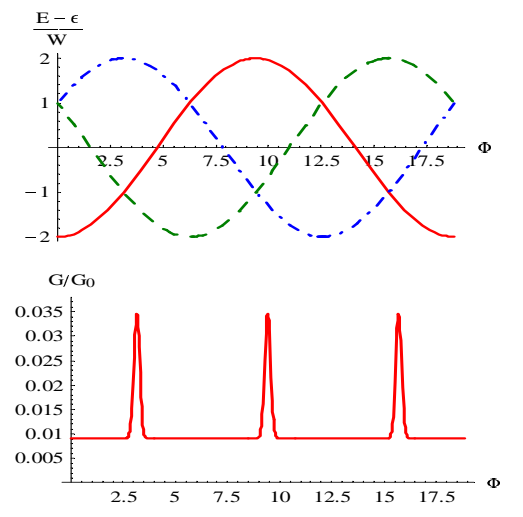

FIG. 10: Upper panel: Evolution of the energy levels $E_{A}$ (solid line) and $E_{ \pm}$(dashed and dashdotted line, resp.) Lower panel: corresponding evolution of conductance $\left(G_{0}=\pi e^{2} / \hbar\right)$.

of TTQD is SU(4), so that the symmetry crossovers $S U(2) \rightarrow S U(4) \rightarrow S U(2)$ occur at $\Phi=\left(n+\frac{1}{2}\right) \Phi_{0}$, where $\Phi_{0}$ is the quantum of magnetic flux. Each crossover is accompanied by the change of $T_{K}$ from $\exp (-1 / 2 J)$ to $\exp (-1 / 4 J)$ and back (see Section III.A). The ZBA peak in the two-terminal tunnel conductance changes accordingly (Fig. 10, lower panel).

Although formally there are three tunneling channels, the non-Fermi liquid Kondo regime cannot arise, because the non-diagonal components $J_{i j}$ appear in the exchange Hamiltonian (14). Further diagonalization should be done by means of rotating frameworks for Bloch electrons. This diagonalization introduces irremovable channel anisotropy, so that the 2channel non-Fermi-liquid fixed point cannot be achieved unlike the case considered in [53], 
where the channel isotropy was postulated from the very beginning.

In order to realize the Aharonov-Bohm interference, one should use the two-terminal geometry shown in Fig. 2d. In this case there are two paths (1-3) and (2-3) for single electron tunneling between source and drain. Interference of two waves in the drain results in famous Aharonov-Bohm oscillations. The field $B$ affects the lead-dot hopping phases. In the chosen gauge, the hopping integrals are modified as, $W \rightarrow W \exp \left(i \Phi_{1} / 3\right), \quad V_{1,2} \rightarrow$ $V_{s} \exp \left[ \pm i\left(\Phi_{1} / 6+\Phi_{2} / 2\right)\right]$, where $\Phi_{1,2}$ are magnetic fluxes through the upper and lower loop of the device. As a result the exchange Hamiltonian reads

$$
H=J_{s} \mathbf{S} \cdot \mathbf{s}_{s}+J_{d} \mathbf{S} \cdot \mathbf{s}_{d}+J_{s d} \mathbf{S} \cdot\left(\mathbf{s}_{s d}+\mathbf{s}_{d s}\right)+K \mathbf{T} \cdot \mathbf{t}
$$

(the latter term becomes actual when the magnetic field induces level crossing in accordance with Fig. 10). Magnetic flux enters the coupling constants $J_{s}, J_{d}, J_{s d}, K$ via SW transformation. As a result the constant $J_{s d}\left(\Phi_{1}, \Phi_{2}\right)$ turns into zero at some values of magnetic flux, so that the Aharonov-Bohm interference completely blocks Kondo transparency. Thus TTQD serves simultaneously as a Kondo "pass valve" and as an Aharonov-Bohm interferometer. It should be stressed that both the continuous spin degrees of freedom and discrete "rotations" of triangle are involved in these two phenomena in TTQD.

\section{CONCLUDING REMARKS}

Among many aspects of Kondo tunneling through complex quantum dots we have chosen

for this review only the symmetry related properties predetermined by the structure of the low-lying states in the spin multiplet characterizing the fixed charge sector of complex quantum dot. New features, which are introduced by the dynamical symmetries in the Kondo physics are the multistage process of Kondo screening, symmetry crossovers driven by experimentally tunable control parameters, interplay between continuous spin rotation symmetry and discrete point symmetry of nanodevices. The main tool of experimental monitoring of variable symmetries is study of temperature and magnetic filed dependence of zero- and finite-bias anomalies in tunnel conductance.

Among other facets of Kondo effect in nanostructures one should mention non-equilibrium Kondo effect at finite bias and under light illumination, where both quantum dots and leads are far enough from thermodynamic balance. Under this conditions such phenomena as 
spin relaxation, dephasing and decoherence influence the tunnel transport in Kondo regime. Real atoms and molecules also may be included in electric circuit by means of advanced experimental techniques (scanning tunnel spectroscopy, break-junction method etc). In this case phonon- and photon assisted processes should be taken into account, which result in interplay of Kondo resonance tunneling with various "polaronic" and "excitonic" effects. Besides, Kondo processes may be included in nano-electro-mechanical shuttling, so that Kondo physics of movable objects becomes one of challenging items on the agenda. 
[1] J. Kondo: Progr. Theor. Phys. 32, 37 (1964)

[2] K.G. Wilson: Rev. Mod. Phys. 47, 773 (1975)

[3] A.M. Tsvelik, P.B. Wiegmann, Advances in Physics, 32453 (1983)

[4] N. Andrei, K. Furuya, J.H. Lowenstein, Rev. Mod. Phys. 55, 331 (1983)

[5] J. Appelbaum, Phys. Rev. Lett. 17, 91 (1966)

[6] P.W. Anderson: Phys. Rev. Lett. 17, 95 (1966)

[7] J.R. Schrieffer, P.A. Wolff: Phys. Rev. 149, 491 (1966)

[8] J. Friedel: Can. J. Phys. 34, 1190 (1956)

[9] P.W. Anderson, Phys. Rev. 124, 41 (1961)

[10] K.S. Dy: Phys. Status Solidi (b) 81, K111 (1977)

[11] B. Coqblin: J.R. Schrieffer, Phys. Rev. 185, 847 (1969)

[12] B. Cornut, B. Coqblin: Phys. Rev. B 5, 4541 (1972)

[13] I. Affleck, J. Marston: Phys. Rev. Lett. 37, 3774 (1988)

[14] N. Read, S. Sachdev: Nucl. Phys. B 316, 609 (1989)

[15] P. Nozieres, A. Blandin: J. Phys. (Paris), 41, 193 (1980)

[16] P. Nozieres: J. Low Temp. Phys. 17, 31 (1974)

[17] A. Zawadowski, K. Vladar: Solid State Commun. 35, 217 (1980)

[18] D.L. Cox: Phys Rev. Lett. 59, 1240 (1987)

[19] S.E. Barnes: Phys. Rev. B 37, 3671 (1988).

[20] D.L. Cox, A. Zawadowski: Adv. Phys. 47943 (1998)

[21] A.K. Zhuravlev, V. Yu. Irkhin, M.I. Katsnelson et al: Phys. Rev. Lett. 93, 236403 (2004).

[22] L.I. Glazman, M.E. Raikh: JETP Lett. 47, 452 (1988)

[23] T.K. Ng, P.A. Lee: Phys. Rev. Lett. 61, 1768 (1988).

[24] D. Goldhaber-Gordon, H. Shtrikman, D. Mahalu et al: Nature 391, 156 (1998)

[25] S.M. Cronenwett, T.H. Oosterkamp, L.P.Kouwenhoven: Science 281, 540 (1998)

[26] F. Simmel, R.H. Blick, J.P. Kotthaus et al: Phys. Rev. Lett. 83, 804 (1999)

[27] K.A. Matveev: Zh. Eksp. Teor. Phys. 98, 1598 (1991) [Sov. Phys. JETP 72, 892 (1991)]; Phys. Rev. B 51, 1743 (1995)

[28] E. Lebanon, A. Shiller: Phys. Rev. B 64, 245338 (2001) 
[29] C.J. Bolech, N. Shah, Phys. Rev. Lett. 95, 036801 (2005)

[30] K. Kikoin, Y. Avishai, Phys. Rev. B 624647 (2000)

[31] M. Pustilnik, Y. Avishai, K. Kikoin, Phys. Rev. Lett. 84, 1756 (2000)

[32] D. Giuliano, A. Tagliacozzo: Phys. Rev. Lett. 84, 4677 (2000)

[33] M. Eto, Yu. Nazarov: Phys. Rev. Lett. 85, 1306 (2000)

[34] Single Charge Tunneling, ed by H. Grabert and M.H. Devoret (Plenum, New York 1992)

[35] Mesoscopic Electron Transport, ed by L.L. Son, L.P. Kouwenhoven and G. Schön (Kluwer, Dordrecht 1997)

[36] W.G. van der Wiel, S. De Franceschi, J.M. Enselman et al: Rev. Mod. Phys. 75, 1 (2003)

[37] F. Hofmann, T. Heinzel, D.A. Wharam et al: Phys. Rev. B51, 13872 (1995)

[38] L.W. Molenkamp, K. Flensberg, M. Kemerlink: Phys. Rev. Lett. 75, 4282 (1995)

[39] C. Livermore, C.H. Crouch, R.M. Westervelt et al: Science 274, 1382 (1996)

[40] J.J. Palacios, P. Hawrilak: Phys. Rev. B 51, 1769 (1995)

[41] D. Loss, D.P. DiVincenzo: Phys. Rev. A 57, 120 (1998)

[42] B. Partoens, F.M. Peeters, Phys. Rev. Lett. 84, 4433 (2000).

[43] K. Kikoin, Y. Avishai: Phys. Rev. Lett. 86, 2090 (2001); Phys. Rev. B 65, 115329 (2002)

[44] N.J. Craig, J.M. Taylor, E.A. Lester et al: Science 304, 565 (2004)

[45] M. Stopa: Phys. Rev, Lett. 88, 146802 (2002)

[46] A. Vidan, R.M. Westervelt, M. Stopa et al: Appl. Phys. Lett. 85, 3602 (2004).

[47] L. Gaudreau, S. Studenikin, A. Sachrajda et al: arXiv: cond-mat/06015967

[48] T. Tanamoto: Phys. Rev. A 61, 022305 (2000)

[49] D.S. Saraga, D. Loss: Phys. Rev. Lett. 90, 166803 (2003).

[50] G. Zaránd, A. Brataas, D. Goldhaber-Gordon: Solid State Commun. 126, 463 (2003)

[51] T. Jamneala et al: Phys. Rev. Lett. 87, 256804 (2001).

[52] B. Lazarovits, P. Simon, G. Zaránd et al: Phys. Rev. Lett. 95, 077202 (2005)

[53] K. Ingersent, A.W. Ludwig, J. Affleck: Phys. Rev. Lett. 95, 257204 (2005).

[54] T. Kuzmenko, K. Kikoin, Y. Avishai: Phys. Rev. Lett. 96, 046601 (2006).

[55] T. Kuzmenko, K. Kikoin, Y. Avishai: Physica E 29, 334 (2005)

[56] C.A. Stafford, S. Das Sarma: Phys. Rev. Lett. 72, 3590 (1994)

[57] C. Klimek, G. Chen, S. Datta: Phys. Rev. B 50, 2316 (1994)

[58] Y. Meir, N.S. Wingreen: Phys. Rev. Lett. 68, 2512 (1992) 
[59] J.M. Golden, B.I. Halperin: Phys. Rev. B 53, 3893 (1996)

[60] D.C. Langreth, Phys. Rev. 150, 516 (1966)

[61] J. Hubbard, Proc. Roy. Soc. A 285, 542 (1965)

[62] M.J. Englefield, Group Theory and the Coulomb Problem (Wiley, New York 1972).

[63] I.A. Malkin, V.I. Man'ko: Dynamical Symmetries and Coherent States of Quantum Systems (Fizmatgiz, Moscow 1979) [in Russian].

[64] K. Kikoin, Y. Avishai, M.N. Kiselev: in Nanophysics, Nanoclusters, Nanodevices (Nova Science, New York 2006)

[65] M.N.Kiselev: Int. J. of Mod. Phys. B, 20, 381 (2006)

[66] T. Ivanov: Europhys. Lett. 40, 183 (1997)

[67] T. Pohjola, J. König, M.M. Salomaa et al: Europhys. Lett. 40, 189 (1997)

[68] T. Aono, M. Eto, K. Kawamura: J. Phys. Soc. Jpn. 67, 1860 (1998)

[69] A. Georges, Y. Meir: Phys. Rev. Lett. 82, 3508 (2002)

[70] V.N. Golovach, D. Loss: Europhys. Lett. 62, 83 (2003)

[71] L. Borda, G. Zarand, W. Hofstetter et al: Phys. Rev. Lett. 90, 026602 (2003)

[72] Y. Tanaka, N. Kawakami: J. Phys. Soc. Jpn. 73, 2795 (2004); Phys. Rev. B 72, 085304 (2005)

[73] R. Sakano, N. Kawakami: Phys. Rev. B 73, 155332 (2005)

[74] M. Eto: J. Phys. Soc. Jpn. 74, 95 (2005)

[75] B.A. Jones, C. Varma: Phys. Rev. B 40, 324 (1989)

[76] W. Hofstatter, H. Schoeller: Phys. Rev. Lett. 88, 016803 (2002)

[77] M. Pustilnik, L.I. Glazman: Phys. Rev. Lett. 85, 2993 (2000); Phys. Rev. B 64, 045328 (2001)

[78] T. Kuzmenko, K. Kikoin, Y. Avishai: Phys. Rev. Lett. 89, 156602 (2002); Phys. Rev. B 69, 195109 (2004)

[79] W. Izumida, O. Sakai, Y. Shimizu: J. Phys. Soc. Jpn. 67, 2444 (1998)

[80] M. Pustilnik, L.I. Glazman, W. Hofstetter: Phys. Rev. B 68, 161303 (2003)

[81] W. Hofstetter, G. Zarand: Phys. Rev. B 69, 235301 (2004)

[82] M. Pustilnik, L.I. Glazman: J. Phys.: Cond. Mat. 16, R513 (2004)

[83] P.W. Anderson: J. Phys. C. Solid State 3, 2436 (1980)

[84] W.G. van der Wiel, S. de Francheschi, T. Fujisawa et al: Science 289, 2105 (2000) 
[85] A.F.Barabanov, K.A.Kikoin, L.A.Maksimov: Teor. Mat. Fiz. 20, 364 (1974)

[86] F.D.M. Haldane: Phys. Rev. Lett. 40, 416 (1978)

[87] A. Kogan, G. Granger, M.A. Kastner et al: Phys. Rev. B 67, 113309 (2003)

[88] K. Kang, S.Y. Cho, J-J. Kim et al: Phys. Rev. B 63, 113304 (2001)

[89] T-S. Kim, S. Hershfield: Phys. Rev. B 63, 245326 (2001)

[90] Y. Takazawa, Y. Imai, N. Kawakami: J. Phys. Soc. Jpn. 71, 2234 (2002)

[91] M.E. Torio, K. Hallberg, A.H. Ceccatto et al: Phys. Rev. B 65, 085302 (2002)

[92] U. Fano: Phys. Rev. 124, 1866 (1961)

[93] A. Shibatani, Y. Toyozawa: J. Phys. Soc. Jpn: 25, 335 (1968)

[94] J. Göres, D. Goldhaber-Gordon, S. Heemeyer et al: Phys. Rev. B 62, 2188 (2000).

[95] M. E. Torio, K. Hallberg, S. Flach et al: Eur. Phys. J. B 37, 399 (2004)

[96] Y. Oreg, D. Goldhaber-Gordon: Phys. Rev. Lett. 90, 136602 (2003)

[97] M. Pustilnik, L. Borda, L.I. Glazman et al: Phys. Rev. B 69, 115316 (2004)

[98] I. Affleck, A.W.W. Ludwig: Phys. Rev. B 48, 7297 (1993)

[99] I. Affleck, A.W.W. Ludwig, H.B. Pang et al: Phys. Rev. B 45, 7918 (1992)

[100] P. Simon, R. Lopez, Y. Oreg: Phys. Rev. Lett. 94, 086602 (2005)

[101] M.G. Vavilov, L.I. Glazman: Phys. Rev. Lett. 94, 086805 (2005)

[102] M. Vojta, R. Bulla, W. Hofstetter: Phys. Rev. B 65, 140405 (2002)

[103] T. Kuzmenko, K. Kikoin, Y. Avishai: Europhys. Lett. 64, 218 (2003)

[104] T. Kuzmenko, K. Kikoin, Y. Avishai: Phys. Rev. B 73, 235310 (2006)

[105] K. Le Hur, P. Recher, E. Dupont et al: Phys. Rev. Lett 96, 106803 (2006)

[106] N. Yu, M. Fowler: Phys. Rev. B 45, 11795 (1992)

[107] J.D. Lee, Phys. Rev. B 67, 153108 (2003)

[108] A. Fuhrer, T. Ihn, K. Ensslin et al: Phys. Rev. Lett. 93, 176803 (2004)

[109] B. Leturcq, L. Schmid, K. Ensslin et al: Phys. Rev. Lett. 95, 126603 (2005)

[110] V.V. Savkin, A.N. Rubtsov, M.I. Katsnelson et al: Phys. Rev. Lett. 94, 026402 (2005)

[111] T. Kuzmenko, K. Kikoin, Y. Avishai: Physica E 29, 334 (2005) 